\title{
La SuPERACIÓN DE LA POBREZA EN CHILE DURANTE EL PRIMER AÑO dEL GOBIERNO dE SEBAstián PIÑERA
}

\author{
Jaime Ruiz-Tagle V. \\ Departamento de Economía y Centro de Microdatos, \\ Universidad de Chile \\ jaimeruiztagle@gmail.com
}

En el trabajo se analiza la implementación de las estrategias destinadas a superación de la pobreza en el primer año del gobierno de Sebastián Piñera. Se argumenta que durante el gobierno que antecedió al actual, se instauró una serie de políticas sociales de carácter asistencialista que apuntaban continuar con la reducción de la pobreza extrema y, por lo tanto, el actual gobierno se encontró con una estrategia de superación de la pobreza de largo plazo que no ha sido mayoritariamente discutida. El Presidente Piñera ha planteado profundizar el carácter asistencialista de la política social en cuestión y el mejoramiento de ciertos aspectos considerados como deficitarios.

Palabras Clave: pobreza, planificación social, políticas públicas, Chile.

\section{OVercoming poverty in ChILE DURING THE FIRST year of the Sebastián Piñera government}

In this paper, poverty reduction strategies implemented during the first year of the Sebastian Piñera government are analyzed. It is argued that the former government implemented a series of welfare-type social policies focused on reducing extreme poverty, while the current government took on this long term strategy without seeming to engage in discussions about new poverty-reduction policies. Indeed, President Piñera has hinted at deepening the welfare-type nature of poverty reduction, while improving on specific aspects deemed unsatisfactory.

Keywords: poverty, social planning, public policy, Chile. 


\section{Introducción}

La superación de la pobreza ha sido un tema prioritario de la agenda social durante los últimos 20 años a lo largo de los gobiernos de la Concertación. Desde el retorno a la democracia, en que los niveles de pobreza se situaban en torno al $40 \%$ se ha planteado como objetivo central de los gobiernos de turno la reducción sistemática de la pobreza y la extrema pobreza o indigencia.

Luego de una reducción significativa de los indicadores de pobreza durante los noventa, el diagnóstico de principios de los 2000 es que la indigencia se ha mostrado más dificil de reducir que la pobreza en general. En efecto, mientras la tasa de pobreza se situaba en 1998 en 22\%, la tasa de indigencia llegaba a 6\%. Es así como surge el programa Puente y luego el programa Chile Solidario. Hacia finales de los 2000 se observa una continuación en la reducción de la pobreza, pero con una velocidad ciertamente menor a lo deseado. La tasa de pobreza a 2009 se situaba en $15 \%$ y la tasa de indigencia en 4\%, mostrando un estancamiento desde 2006.

Durante el gobierno de la Presidenta Bachelet, se instauró de manera profunda una serie de políticas sociales de carácter asistencialista que apuntaban justamente a continuar con la reducción de la pobreza extrema. El gobierno del Presidente Piñera se encontró entonces con una estrategia de superación de la pobreza de largo plazo que no ha sido mayoritariamente discutida. Por el contrario, desde su inicio el gobierno del Presidente Piñera ha planteado profundizar el carácter asistencialista de la política social en cuestión y el mejoramiento de ciertos aspectos considerados como deficitarios.

\section{Compromisos explicitados por el gobierno de Sebastián Piñera}

El plan de Superación de Pobreza del gobierno del Presidente Piñera contiene los siguientes objetivos:

- Derrotar la pobreza extrema en el 2014 y erradicar la pobreza antes del año 2018.

- Rediseño global de la política contra la pobreza, para producir una articulación efectiva de programas, mayor celeridad en la identificación de beneficiarios y simplificación de la entrega de los beneficios.

- Dar más opciones a la gente de menores recursos, a través de programas de subsidio a las personas, de manera que tengan más alternativas para elegir. 
- Perfeccionamiento de la Ficha de Protección Social (FPS), para que las personas que necesitan apoyo no queden excluidas por razones políticas o por otras discriminaciones injustas.

- Incentivar la participación de la Sociedad Civil, dado que ella ha probado ser particularmente eficiente en el combate contra la pobreza, en particular en los núcleos de pobreza dura.

Los mecanismos identificados para cumplir los objetivos son los siguientes:

Fortalecimiento del Ministerio de Planificación -MIDEPLAN- a través de la creación del Ministerio de Desarrollo Social, funcionará en La Moneda bajo la directa supervisión del Presidente de la República. El nuevo ministerio quedará a cargo del "componente social» del actual MIDEPLAN: caracterización socioeconómica de la población (CASEN, FPS, SIIS) y coordinación de los fondos y servicios relacionados con los grupos más vulnerables de la población. En este rol, el ministerio ampliará y perfeccionará el programa Puente y los programas Chile Solidario y Chile Crece Contigo.

La creación de un Ingreso Ético Familiar, cuyos componentes serán el Subsidio Único Familiar, un Bono de Capacitación y un Subsidio al Trabajo. Este Ingreso Ético Familiar irá acompañado de algunos requerimientos para acceder a él, tales como asistencia escolar, control de salud, esfuerzos de capacitación y búsqueda de empleo. En el caso del subsidio por niño, éste se aumentará significativamente. Existirá un mecanismo gradual de retiro de las transferencias recibidas por los grupos beneficiarios, con el fin de evitar que el hogar pierda los incentivos a abandonar su condición de vulnerabilidad. A esto se suman los anuncios del 21 de mayo referidos al Bono de Intermediación Laboral para trabajadores de baja calificación vulnerables y el perfeccionamiento del Seguro de Cesantía.

Se entregará el "Bono de Garantía Auge", que permita a los usuarios escoger con mayor libertad el hospital público o privado de su preferencia, cuando no se cumplan los plazos garantizados por el Auge; una subvención de educación preescolar en función de requisitos como asistencia; se otorgará un «Bono de Capacitación» y un «Bono de Asistencia Judicial», este último concebido para que personas pobres puedan pagar un abogado cuando las Corporaciones Judiciales que existen para tal efecto no los atiendan adecuadamente.

Promover una alianza público-privada para superar la pobreza dura, a través de la creación de un mecanismo mediante el cual el Estado suplemente los aportes privados a 
instituciones de la sociedad civil dedicadas a trabajar con la población en condiciones de pobreza extrema, en un monto similar al aportado por las entidades privadas.

Paralelamente, existe una serie de compromisos que, si bien se relacionan más directamente con el mercado laboral, pueden afectar de forma significativa la superación de la pobreza. Dentro de estos compromisos se cuentan:

- Bono para que cada trabajador pueda decidir dónde y en qué capacitarse.

- Bono de intermediación laboral para personas de baja calificación vulnerables.

- Bolsa Nacional de Empleo.

- Perfeccionamiento del seguro de desempleo.

Finalmente, el ministro Kast relanzó durante abril la Agenda Social centrada en cuatro iniciativas: las Bodas de Oro; la Reducción Gradual del 7\% de Salud para jubilados; la extensión del Post Natal y el pago de la Asignación Social.

\section{Estado o realidad de avance}

\section{Aspectos relevantes en la Ley de Presupuestos}

La Ley de Presupuesto enviada al Congreso contempla una serie de elementos relacionados directamente e indirectamente con la superación de la pobreza. A continuación se revisan algunos aspectos vinculados al presupuesto de las principales partidas presupuestarias de Mideplan y de algunos programas del Ministerio del Trabajo.

El Programa Chile Solidario contempla un presupuesto de M\$160.872.193 para el 2011 , lo que implica un incremento real ${ }^{1}$ de $73 \%$ respecto a 2010 (M\$90.259.089). La principal alza se debe a la inserción del Programa Bonificación al Ingreso Ético Familiar, que tiene contemplado un monto de $M \$ 64.046 .000$. Se establece también que "con la finalidad de mejorar significativamente el impacto del componente empleo de este programa, todos los beneficiarios del Sistema Chile Solidario tendrán acceso preferente en todos los programas de generación de empleo previstos en esta Ley de Presupuesto”. Ello incluye:

a) $\mathrm{M} \$$ 7.309.146 para la implementación del componente de apoyo psicosocial del Sistema Chile Solidario.

El deflactor implícito del presupuesto es de 3.3\%. 
b) M\$10.561.545 para la ejecución de líneas o programas de apoyo al microemprendimiento.

c) M\$ 720.527 para el Programa de Apoyo a la Inserción Laboral Juvenil.

d) $M \$ 2.066 .000$ para el Programa "Preparación para el Trabajo".

Un cálculo simple de los montos involucrados en el Ingreso Ético Familiar indica que, dadas las aproximadamente 350 mil familias del Chile Solidario, con aproximadamente 5 personas por hogar, podrían recibir como máximo una suma de unos $\$ 3.000$ mensuales por persona (aproximadamente un 5\% de la línea de pobreza). Con esto, se puede esperar que este componente tenga un impacto bastante acotado en reducción de pobreza. Sin embargo, si los beneficios se lograran focalizar completamente en las familias en situación de indigencia (pobreza extrema), se podría tener efectos más importantes. Si bien la focalización en dicho grupo ha demostrado ser extremadamente compleja, dado que ello implicaría cubrir a unas 85 mil familias, se podría tener impactos relevantes en reducción de pobreza extrema (se tendrían unos $\$ 12.500$ mensuales per cápita, equivalente a un $40 \%$ de la línea de indigencia urbana).

Por su parte, el reordenamiento a través del nuevo Ministerio Social, si bien deseable, difícilmente producirá impactos de corto o mediano plazo en los niveles de pobreza. No obstante, en la búsqueda de una mayor eficiencia en el diseño, evaluación e implementación de las políticas sociales, es esperable observar impactos de largo plazo.

El Sistema de Protección Integral a la Infancia (Chile Crece Contigo) contempla en la Ley de Presupuestos del año 2011 un monto de $M \$ 37.056 .152$. Esto corresponde a un alza de un 5\% real con respecto al presupuesto del año 2010 (M\$34.116.960). Se avizora entonces una política de continuidad del programa.

Paralelamente, hay una serie de temáticas vinculadas a la problemática de la pobreza que dicen relación con el mercado laboral y la formalidad. En efecto, en la Ley de Presupuesto se contemplan los siguientes ítems:

- Bono de capacitación: Consiste en un bono especial para que cada trabajador pueda decidir dónde y en qué capacitarse.

- Capacitación sobre Teletrabajo: Consiste en la capacitación a empresarios y trabajadores acerca de beneficios del Teletrabajo. Se utilizarán las tecnologías digitales disponibles, que facilitaría esta nueva modalidad de trabajo. 
- Bono de Intermediación Laboral: Este bono, financiando por el Estado, tiene como fin ayudar a encontrar trabajo, a personas de baja calificación, a través de agencias dedicadas a buscar trabajos estables para personas vulnerables.

- Bolsa Nacional de Empleo: Pretende que más de 7 millones de trabajadores conozcan mejores opciones laborales.

- $\quad$ Seguro de desempleo: Se perfeccionará el seguro de desempleo, para que más trabajadores puedan acceder a él y queden debidamente protegidos.

Adicionalmente, la Ley de Presupuesto contempla incrementar los recursos del SENCE con el objetivo de aumentar la capacitación en micro, pequeñas y medianas empresas, integrando los nuevos recursos del SENCE al sistema de educación técnico profesional.

\section{Estado de avance de los compromisos adquiridos}

La implementación del Ministerio de Desarrollo Social se encuentra en Primer Trámite Constitucional en la Cámara del Senado con plazo para presentar indicaciones hasta el 22 de marzo. Por su parte, la implementación del Ingreso Ético Familiar ha avanzado con la implementación de lo que se ha llamado la "Asignación Familiar". Es así como a partir del 1 de abril de este año, regirá la implementación de la primera etapa del proyecto que consiste en una entrega mensual de un bono de 7.500 pesos por cada integrante de la familia además de los 5.000 pesos por hijo a las $130 \mathrm{mil} \mathrm{fa-}$ milias más vulnerables del programa Chile Solidario, hasta el 31 de diciembre de 2011.

En cuanto a los compromisos relacionados con el mercado laboral se tiene:

- Bono para que cada trabajador pueda decidir dónde y en qué capacitarse. Estado actual: Se anuncia extensión nacional del Bono de Capacitación.

- Bono de intermediación laboral para personas de baja calificación vulnerables. Estado actual: Se presenta en Arica Bono de Intermediación Laboral.

- $\quad$ Bolsa Nacional de Empleo. Estado: Implementado.

- Perfeccionamiento del seguro de desempleo. Estado: No hay avances al respecto.

- Por su parte, la implementación de los programas sociales que vienen de la administración anterior ha seguido su curso de manera bastante sostenida, sin haber grandes cambios respecto a la situación de 2009. 


\section{Evaluación global}

\section{Visión general de las políticas de reducción de pobreza}

Uno de los desafios del país en los próximos años es continuar el avance en reducción de la pobreza y en particular de la extrema pobreza. El diagnóstico de los últimos años indica que la reducción de la pobreza ha entrado en una etapa más lenta producto principalmente de la naturaleza de la problemática misma.

Los objetivos planteados por el gobierno actual no son muy distintos a los planteados por los gobiernos anteriores y se sustentan fuertemente en un esperado crecimiento económico sostenido por los próximos cuatro años. En efecto derrotar la extrema pobreza en 2014 se presenta como un desafío en extremo complejo por cuanto la extrema pobreza ha demostrado ser no sólo dificil de focalizar, sino que además poco sensible a las políticas sociales asistenciales y no asistenciales.

Por otro lado, el objetivo de rediseñar la política de pobreza aparece como un objetivo interesante, aunque prácticamente imposible de evaluar si no es a través de los resultados de reducción de pobreza. Por su parte, se sugiere utilizar mecanismos de subsidios a las personas, lo que no es muy distinto a los mecanismos de protección social del gobierno anterior.

Por su parte, el perfeccionamiento de los instrumentos de focalización de las políticas sociales ha sido parte de las acciones de los gobiernos anteriores, aunque ciertamente debería constituir un proceso continuo. Asimismo, el objetivo de incentivar la participación de la sociedad civil constituye un concepto en extremo amplio, por lo que dificilmente se podrán establecer avances cuantitativos en la materia.

\section{Políticas laborales y combate a la pobreza}

Las políticas laborales debieran estar orientadas esencialmente a generar condiciones de empleo, aumentar la productividad de los trabajadores, y facilitar la velocidad de ajuste del mercado laboral ante cambios macroeconómicos y/o sectoriales. Ciertamente las políticas de empleo tienen impacto en la situación de pobreza de la población. Sin embargo, las políticas laborales deben estar orientadas a todo el mercado del trabajo y no necesariamente hacerse cargo de las carencias de ingreso de los hogares.

La utilización del mercado laboral para resolver temas de pobreza tiene efectos limitados (por cobertura debido a informalidad y políticas que sólo llegan a empresas de pequeñas hacia arriba). Además, se corre el riesgo de generar distorsiones complejas en el mercado laboral. Las políticas sociales que sí están orientadas directamente a 
afectar pobreza no debieran pasar de manera directa por el mercado laboral, aunque sí es deseable que se utilicen mecanismos complementarios.

\section{Pobreza crónica vs. pobreza transitoria}

La pobreza crónica es multidimensional y es poco sensible a la dinámica laboral. La pobreza extrema ha mostrado ser bastante insensible a la oferta pública establecida, donde los programas existentes no se adecuan particularmente a sus necesidades. Este tipo de pobreza requiere un fuerte componente asistencial. Ni siquiera los países ricos han logrado erradicarla. La extensión de la informalidad laboral hace que las políticas laborales tengan serios problemas de cobertura.

La pobreza transitoria (o circunstancial) obedece a situaciones transitorias relacionadas con shocks de empleo y/o de necesidades (shocks de salud o reestructuración de hogares). La pobreza transitoria está muy relacionada con los ciclos económicos y con las transformaciones de ciertos sectores económicos, donde las políticas de empleo pueden jugar un rol importante: seguro de cesantía, programas de empleo de emergencia, programas de reinserción y reconversión laboral. El aumento de la participación laboral femenina juega un rol fundamental como amortiguador de los shocks de empleo. La flexibilidad del mercado laboral para ajustarse vía salarios y/o número de horas trabajadas, en vez de hacerlo vía empleo, es fundamental para evitar la pobreza transitoria.

\section{Lo que contiene la Ley de Presupuesto}

Se plantea una agenda "pro-empleo" con una serie de modificaciones e innovaciones de programas laborales contenidas en la Ley de Presupuesto. El Ingreso Ético Familiar y su vínculo con las políticas de empleo se reduce a las mujeres y el empleo formal a través de subsidios al empleo.

Los programas de capacitación nuevos (Bono de Capacitación, Bono de Capacitación Trabajadores Activos, Programa de Capacitación en Oficios, y Programa de Formación en el Puesto de Trabajo) vienen más bien a sustituir a los programas existentes anteriormente.

Hay un fuerte componente de subsidio al empleo, pero ello no asegura que tenga un impacto en pobreza al no estar directamente vinculado de la situación de pobreza de los trabajadores (sólo condicionado al nivel de salario). De hecho, está canalizado a través de las empresas y no a través de los individuos. 


\section{Una pieza faltante: Programas Welfare to work (de los subsidios al empleo)}

Los programas Welfare-to-work se relacionan con ayudar a los beneficiarios a dejar los beneficios del Estado y hacerse auto-suficientes (a través del cuidado infantil, entrenamiento para el empleo y asistencia laboral). La esencia de estos programas es premiar la condición de empleo, con el objeto de desincentivar, en términos relativos, la sobreutilización de los subsidios. Experiencias en países desarrollados principalmente en Estados Unidos y Reino Unido.

¿Qué han aprendido en Estados Unidos?:

- Programas suplementarios de ingresos son eficientes como mecanismo de transferencia porque los beneficiarios reciben más que lo que le cuesta al Estado.

- Programas que condicionan los beneficios a la condición de ocupación en el corto y largo plazo son costo-efectivos para beneficiarios y para el Estado.

- Programas que condicionan los beneficios a la búsqueda de empleo son más baratos, pero menos eficientes.

- Programas de capacitación previa no generan mayor impacto para los beneficiarios.

Algunos ejemplos de programas de empleo de gobierno en Estados Unidos: Workertrainee: programa de aprendices en empleos públicos, donde los beneficiarios "ganan dinero mientras aprenden". Duran tres años “a prueba”, antes de entrar como funcionarios de carrera; Veterans' Preference: acceso preferencial a empleos públicos. Job Corps: programa académico, vocacional y de habilidades sociales para jóvenes vulnerables.

Por su parte, la experiencia en el Reino Unido nos permite identificar algunos ejemplos: Work for Your Benefit provee seis meses de empleo de emergencia; New Deal for Lone Parents y Working Families' Tax Credit entregan subsidios complementarios a quienes trabajan al menos 16 hrs a la semana. Estas políticas han sido eficientes, con impactos de largo plazo, aunque moderados (hasta siete puntos porcentuales de empleo).

\section{Discusión final}

El fortalecimiento de Mideplan a través de la creación de un Ministerio de Desarrollo Social constituye un elemento importante que ha estado en la agenda de los 
gobiernos durante varios años. En efecto, el diagnóstico de lo inapropiado que resulta tener en una misma institución el diseño, ejecución y evaluación de las políticas sociales existe desde hace más de un lustro. No obstante, el funcionamiento del nuevo ministerio es aún una incógnita, de modo que no es posible llevar a cabo a la fecha una evaluación de este aspecto metodológico.

La implementación definitiva, más allá de la Asignación Familiar, del Ingreso Ético Familiar se avizora como una revisión y un reordenamiento de los subsidios y bonificaciones existentes. El impacto efectivo que pueda tener en la situación de pobreza de las personas dependerá críticamente de las condiciones que se establezcan para acceder a cada uno de los componentes del programa.

El bono de garantía Auge no parece estar orientado a aumentar la cobertura del sistema frente a grandes gastos de salud. Por el contrario, pareciera que se pretende introducir un cambio metodológico orientado a obtener eventuales mejoramientos de eficiencia a través de una mayor utilización de la oferta privada en desmedro de la pública. Aunque se desconocen detalles, se puede prever que el impacto en materia de pobreza de este aspecto será limitado.

La promoción de alianzas público-privadas para contribuir a superar la pobreza extrema aparece como una innovación metodológica que llama la atención por la extensión del impacto que pueda tener. Si bien se puede pensar que organizaciones con cercanía a las personas en situación de pobreza y de pequeña escala pueden tener diagnósticos y ejecuciones interesantes en términos de impacto sobre la pobreza, la evidencia al respecto es cuasi nula. Aunque es difícil argumentar que pequeñas instituciones no gubernamentales estén llevando a cabo trabajos sin impacto, tampoco se puede afirmar lo contrario, y menos aún que sea factible replicarlos y/o escalarlos. Por su parte, la creación de las alianzas con los privados deberá ser evaluada en virtud de los incentivos que puedan tener los privados de participar en este tipo de acciones una vez que se conozcan más detalles de los proyectos.

Por su parte, una revisión de los probables impactos en pobreza de los programas laborales indica que aquellos vinculados a capacitación pueden tener un impacto bastante limitado en el corto plazo sobre la empleabilidad de las personas y sobre sus ingresos. No obstante, la intermediación laboral, la bolsa nacional de empleo y el mejoramiento del seguro de desempleo sí podrían tener un mayor impacto en breve plazo sobre la pobreza. 
La bonificación para la intermediación laboral supone la existencia en el mercado de instituciones adecuadas que ayuden efectivamente a colocar a los trabajadores, lo que muy probablemente es un supuesto temerario. La Bolsa Nacional de Empleo surgió durante la segunda parte del gobierno anterior y va en la misma línea. Los trabajadores más vulnerables serán probablemente quienes menos puedan aprovechar estos programas.

El fortalecimiento del seguro de desempleo es un anhelo de cierta data, y la Ley de Presupuesto enviada no contribuye, por ahora, a esclarecer cómo se intentará mejorar el sistema y aumentar los beneficios a más trabajadores.

En suma, los programas asociados al SENCE tienen una clara connotación de subsidio a través de las empresas. De hecho, en la ejecución de 2010 ya se observa tal tendencia: en 2010 hay 4.038 empresas beneficiadas y 41.238 contratos, mientras que en 2009 fueron 2.708 empresas y 24.104 contratos.

La política laboral entonces busca generar empleo formal, lo cual es ciertamente deseable, pero es probable que poco pueda hacer en la dirección de generar empleo para los más vulnerables. En efecto las especiales características de vulnerabilidad de la población en extrema pobreza han mostrado ser bastante insensibles a la oferta pública establecida, donde los programas existentes no se adecuan particularmente a sus necesidades. Por ello, la política asistencial en que se busque asistir a los más vulnerables jugará un rol fundamental, y por ahora al menos no se observa un énfasis presupuestario en ello.

\section{Evaluación de los programas insignes}

\section{INGRESO ÉTICO FAMILIAR}

\section{Anuncio Presidencial}

El Presidente Piñera, anunció "la primera etapa del Ingreso Ético Familiar”, como "el punto de partida de una serie de medidas que se impulsarán buscando superar la pobreza extrema para el año 2014”. El anuncio indica que el programa regirá “a partir del 1 de abril de este año y se entregará mensualmente a las 130.000 familias más vulnerables del programa Chile Solidario (aproximadamente 490.000 personas), desde el 1 de abril, hasta el 31 de diciembre de 2011, según su estado de vulnerabilidad:"

- Grupo 1: \$7.500 por cada integrante del grupo familiar. 
- Grupo 2: \$6.000 por cada integrante del grupo familiar.

- Grupo 3: $\$ 4.500$ por cada integrante del grupo familiar.

Se indica además que "serán beneficiarios de esta bonificación todas las familias que en 2011 sean miembros de Chile Solidario; reciban bonos de protección, egreso o apoyo psicosocial y tengan un puntaje menor a 4.213 puntos en su Ficha de Protección Social."

Adicionalmente, el proyecto considera tres beneficios condicionados que se suman a los anteriores:

1. Beneficio Adicional por Escolaridad: A las familias con hijos entre 6 y 18 años, que estén matriculados en establecimientos educacionales reconocidos oficialmente por el Ministerio de Educación, para cursar Educación Básica o Media. Por cada niño matriculado, el grupo más vulnerable recibirá $\$ 5.000$ más al mes. Luego, si esos niños cumplen con una asistencia del $85 \%$ percibirán otros $\$ 5.000$.

2. Beneficio Adicional Control de Niño Sano:A familias con hijos de edad inferior a 6 años al 31 de marzo de 2011, con el carnet de Control de Salud del niño o niña al día recibirán $\$ 5.000$. En el segundo grupo de vulnerabilidad este beneficio será de $\$ 4.000$ y en el caso del tercer grupo, que está a punto de dejar la pobreza extrema, baja a $\$ 3$ mil.

3. Beneficio Adicional por Inserción Laboral de La Mujer: A las mujeres integrantes de las familias seleccionadas, mayores de 18 años, que no registren cotizaciones previsionales entre abril de 2009 y marzo de 2011 y que tengan un mínimo de tres cotizaciones previsionales entre abril a octubre de este año, recibirán un aporte por este concepto con un tope de $\$ 51.600$.

\section{Análisis del diseño}

La implementación del Ingreso Ético Familiar se basa en el programa Chile Solidario (CHS) y en la Ficha de Protección Social (FPS), por lo que no conlleva un rediseño de los mecanismos de focalización existentes. Si bien no hay evidencia que el CHS sea un programa particularmente exitoso en la superación de la extrema pobreza, sí ha sido muy criticado por el gobierno actual al presentar las cifras de pobreza de Casen 2009. Por otro lado, la evidencia muestra que la FPS tiene serios problemas en identificar adecuadamente la pobreza monetaria. Resulta entonces llamativo que el proyecto del Gobierno actual no presente innovaciones de diseño al respecto. 
El proyecto del Ingreso Ético Familiar presenta dos componentes fundamentales: bonos e incentivos al trabajo. Los bonos ya estaban contenidos en las políticas sociales del gobierno anterior, mientras que los incentivos al trabajo formaban parte fundamental de las recomendaciones de la Comisión Asesora Presidencial sobre Trabajo y Equidad. De esta manera, en términos de diseño no se encuentran elementos novedosos.

El componente de incentivos es limitado, tanto en su cobertura como en su intensidad. La proporción de la población con "controles de niño sano" al día es lo suficientemente alta como para no ser relevante en la posibilidad de generar un cambio de comportamiento. Lo mismo ocurre con la matrícula y la asistencia escolar.

Por otro lado, el incentivo al trabajo formal para las mujeres es de una cobertura previsiblemente baja. En efecto, la proporción de mujeres que trabaja en el décil de ingresos más bajo es inferior al 20\%, y de ellas el trabajo formal no superaría el 30\%. Por lo tanto, el efecto agregado de este incentivo no tendría una cobertura mayor al $7 \%$ de las mujeres en situación de extrema pobreza.

La implementación del proyecto presenta grandes desafios que no son abordados en el diseño del programa. La información de asistencia escolar que se maneja actualmente corresponde a la del año anterior, de modo que no es útil para la entrega de beneficios por un comportamiento actual. Más aún, aunque se contara con información al día, la entrega de beneficios siempre estaría ocurriendo con un rezago significativo.

Efectos de largo plazo

Existe un consenso importante (plasmado en el informe de la Comisión de Trabajo y Equidad) en que la superación de la pobreza en el largo plazo solo es posible a través del empleo. Lo anteriormente expuesto muestra que el proyecto no propone una salida a la extrema pobreza que vaya más allá de la transferencia monetaria de recursos. Por lo tanto, persisten dudas acerca de la pertinencia del diseño general del proyecto.

Paralelamente, una tarea pendiente de las políticas sociales es el perfeccionamiento de los mecanismos de focalización, que no solo permitan asegurar que las personas que más requieren los beneficios de los programas sean quienes efectivamente se beneficien, sino que además se aseguren la eficiencia en el gasto social. Para ello es fundamental avanzar en mecanismos de salida de los beneficios. Es decir, en mecanismos que hagan que una persona deje de percibir beneficios a medida que progresa económicamente. La implementación de la Pensión Básica Solidaria (PBS) 
ya ha dado muestras de problemas en este sentido. El proyecto del Ingreso Ético Familiar contempla un mecanismo de congelamiento del puntaje FPS al momento de entrar al programa, de modo que una persona que comienza a recibir el beneficio tendría un beneficio adquirido para siempre. La formulación de las políticas sociales debería dar un paso adelante en focalización, y el proyecto en cuestión no solo no innova, sino que profundiza los problemas actuales.

\section{BONO BODAS DE ORO}

Paralelamente, se ha comenzado la implementación del Bono Bodas de Oro, de 250 mil pesos por matrimonio, que "se pagará en partes iguales a cada uno de los cónyuges y beneficiará a aquellas parejas que cumplan 50 años de casados entre el 1 de enero de 2010 y el 31 de diciembre de 2013 y beneficiará al 60\% más vulnerable de la población de acuerdo a la ficha de protección social". Este programa adolece de problemas similares a los del Ingreso Ético Familiar. En primer lugar, no innova en mecanismos de focalización. En segundo lugar, ni siquiera se focaliza en la pobreza extrema. De hecho, dado que el programa beneficiará a personas de al menos 68 años, sería bastante más pertinente utilizar el instrumento de focalización previsional para la PBS que se implementó en 2010, el cual corrige parcialmente problemas de focalización por la forma en que se consideran los ingresos de las personas mayores de 65 años.

\section{OTRAS POLÍTICAS SOCIALES PARA LA SUPERACIÓN DE LA POBREZA}

Hay otras políticas sociales en curso que podrían tener un impacto en pobreza. En efecto, el Subsidio al Empleo Joven, que viene de la administración anterior, tiene un impacto potencial importante en la medida que jóvenes de hogares pobres logren incorporarse al trabajo formal. El programa tiene una gran cobertura e involucra grandes recursos fiscales. Hay una evaluación en curso del impacto del programa, pero por ahora es dificil anticipar resultados. No obstante, dicho programa no ha innovado en sus mecanismos de focalización, por lo que su impacto en pobreza extrema se avizora como de segundo orden.

Del mismo modo, FOSIS ha continuado con el Programa de Apoyo al Microemprendimiento (PAME), que por más de 6 años ha capacitado a más 100 mil microemprendedores. Hay una evaluación en curso desde 2009 de dicho programa que podrá dar luces de su impacto. No obstante, nuevamente se extraña una preocupación 
manifiesta por mejorar la focalización del PAME. La superación de la pobreza extrema requiere de manera urgente seguir avanzando en esta dirección.

Finalmente, el Presidente Piñera anunció que antes de fin de mayo será enviado al Congreso el Proyecto de Ley que elimina o reduce el 7\% que se descuenta a los pensionados por concepto de salud. Este cambio en la ley, orientado a sectores más vulnerables y de clase media, generará un aumento de las pensiones, con cargo directo al Estado que tendrá que proveer al sistema de salud los recursos faltantes. El impacto en pobreza se avizora como limitado. Esto es porque, luego de la implementación de la PBS, la pobreza extrema en los adultos mayores prácticamente se superó.

\section{Referencias}

http://www.gobiernodechile.cl/especiales/plan-de-reconstruccion-2010-2013/

http://www.mideplan.cl/index.php?option $=$ com_content $\& v i e w=\operatorname{article} \& i d=576$ 0:mideplan-ya-echo-a-andar-plan-de-accion-para-superar-la-pobreza-en-losrios\&catid $=213$ : region-rios\&Itemid $=76$

http://www.gobiernodechile.cl/programa-de-gobierno/seguridades/superacion-de-lapobreza/ 